Pacific Journal of Mathematics

TRANSCENDENTAL CONSTANTS OVER THE COEFFICIENT
FIELDS IN DIFFERENTIAL ELLIPTIC FUNCTION FIELDS 


\title{
TRANSCENDENTAL CONSTANTS OVER THE COEFFICIENT FIELDS IN DIFFERENTIAL ELLIPTIC FUNCTION FIELDS
}

\author{
KEIJI NiSHIOKA
}

Let $k$ be a differential field of characteristic 0 , and $\Omega$ be a universal extension of $k$. Suppose that the field of constants $k_{0}$ of $k$ is algebraically closed. Consider the following differential polynomial of the first order over $k$ in a single indeterminate $y$ :

$$
T(y)=\left(y^{\prime}\right)^{2}-\lambda S(y ; \kappa) ; \quad \lambda \in k ; \quad \lambda \neq 0 ;
$$

here

$$
\begin{aligned}
& S(y ; \kappa)=y(1-y)\left(1-\kappa^{2} y\right) ; \\
& \quad \kappa \in k ; \quad \kappa^{2} \neq 0,1 ; \quad \kappa^{\prime}=0 .
\end{aligned}
$$

Take a generic point $z$ of the general solution of $T$. Then, $z$ is transcendental over $k$, and $k\left(z, z^{\prime}\right)$ is called a differential elliptic function field.

We prove the following:

Theorem. Let $k\left(z, z^{\prime}\right)$ be a differential elliptic function field over $k$. Then, there exists a finitely generated differential extension field $k^{*}$ of $k$ such that the following three conditions are satisfied:

(i) $z$ is transcendental over $k^{*}$;

(ii) the field of constants of $k^{*}$ is the same as $k_{0}$;

(iii) there exists an element $\zeta$ of $\Omega$ such that $k^{*}\left(z, z^{\prime}\right)=$ $k^{*}\left(\zeta, \zeta^{\prime}\right)$ and $\left(\zeta^{\prime}\right)^{2}=4 S(\zeta ; \kappa)$ with the same modulus as $\kappa$.

Matsuda [3] gave an example of a differential elliptic function field such that $k=\bar{k}$ and we can not take $k$ as $k^{*}$ (cf. [5]).

REMARK. Matsuda [3] gave a differential algebraic proof of the following theorem essentially due to Poincaré: Suppose that a differential algebraic function field $K$ over an algebraically closed coefficient field $k$ is free from parametric singularities. Then, $K$ is a differential elliptic function field over $k$ if the genus of $K$ is 1 .

The author wishes to express his sincere gratitude to Dr. M. Matsuda who presented this problem and gave kind advices.

1. Two lemmas, The following theorem is due to Kolchin [1]:

LemMA 1. Let $\Sigma$ be a perfect differential ideal in the differential polynomial algebra $k\{y\}$, and let $J$ be a differential polynomial 
in $k\{y\}$ which is not in $\Sigma$. Then, $\Sigma$ has a zero $\eta$ in $\Omega$ such that $J(\eta) \neq 0$ and the field of constants of $k\langle\eta\rangle$ is $k_{0}$.

We shall prove the following:

Lemma 2. Let $F$ be an element of $k\{y\}$ of the first order and let $\xi$ be a zero of $F$ which is transcendental over k. Suppose that $F$ is algebraically irreducible over the algebraic closure $\bar{k}$ of $k$, and that the field of constants of $k\langle\xi\rangle$ is $k_{0}$. Then, there exists a nonsingular zero $\eta$ of $F$ such that $\xi$ is transcendental over $k\langle\eta\rangle$ and the field of constants of $k\langle\eta\rangle$ is $k_{0}$.

Proof. Let $\eta$ be a generic point of the general solution of $F$ over $\overline{k\langle\xi\rangle}$. Then, $\eta \notin \overline{k\langle\xi\rangle}$ and $\eta \notin \bar{k}$. Hence, $\xi \notin \overline{k\langle\eta\rangle}$. By Gourin's theorem (cf. [4, p. 49]) both $\xi$ and $\eta$ are generic points of the general solution of $F$ over $k$. Hence, there exists an isomorphism of $k\langle\xi\rangle$ onto $k\langle\eta\rangle$ over $k$. Therefore, the field of constants of $k\langle\eta\rangle$ is $k_{0}$.

2. Proof of Theorem. We shall prove that there exists a nonsingular zero $w$ of $T$ such that $z$ is transcendental over $k\langle w\rangle$ and the field of constants of $k\langle w\rangle$ is $k_{0}$. First we shall assume that the field of constants of $k\langle z\rangle$ contains properly $k_{0}$. Let $\Sigma$ be the prime differential ideal in $k\{y\}$ associated with the general solution of $T$. Then, the separant $2 y^{\prime}$ of $T$ does not belong to $\Sigma$. By Lemma 1 , there exists a nonsingular zero $w$ of $T$ such that the field of constants of $k\langle w\rangle$ is $k_{0}$. Suppose that $z$ is algebraic over $k\langle w\rangle$. Then, the field of constants of $k\langle z\rangle$ is contained in $k_{0}$, since $k\langle z\rangle \subseteq \overline{k\langle w\rangle}$. This contradicts our assumption. Hence, $z$ is transcendental over $\overline{k\langle w\rangle}$. Secondly, let us assume that the field of constants of $k\langle z\rangle$ is the same as $k_{0}$. Then, there exists a nonsingular zero $w$ of $T$ such that the field of constants of $k\langle w\rangle$ is $k_{0}$ and $z$ is transcedental over $k\langle w\rangle$ by Lemma 2 , since $T$ is algebraically irreducible over $\bar{k}$.

We shall denote $k\langle w\rangle$ by $k_{1}$. Let us define an element $a$ of $k_{1}\langle z\rangle$ by

$$
a=\left\{B(z, w)-2 \lambda^{-1} w^{\prime} z^{\prime}\right\} / A(z, w)^{2},
$$

where

$$
\begin{aligned}
& A\left(y_{1}, y_{2}\right)=1-\kappa^{2} y_{1} y_{2} \\
& B\left(y_{1}, y_{2}\right)=y_{1}\left(1-y_{2}\right)\left(1-\kappa^{2} y_{2}\right)+y_{2}\left(1-y_{1}\right)\left(1-\kappa^{2} y_{1}\right)
\end{aligned}
$$

The polynomials $A, B$ and $S$ satisfy a relation:

$$
B\left(y_{1}, y_{2}\right)^{2}=4 S\left(y_{1}\right) S\left(y_{2}\right)+\left(y_{1}-y_{2}\right)^{2} A\left(y_{1}, y_{2}\right)^{2}
$$


which is verified in the following:

$$
\begin{aligned}
B\left(y_{1},\right. & \left.y_{2}\right)^{2}-4 S\left(y_{1}\right) S\left(y_{2}\right) \\
& =\left\{y_{1} y_{2}^{-1} S\left(y_{2}\right)+y_{2} y_{1}^{-1} S\left(y_{1}\right)\right\}^{2}-4 S\left(y_{1}\right) S\left(y_{2}\right) \\
& =\left\{y_{1} y_{2}^{-1} S\left(y_{2}\right)-y_{2} y_{1}^{-1} S\left(y_{1}\right)\right\}^{2} \\
& =\left\{y_{1}\left(1-y_{2}\right)\left(1-\kappa^{2} y_{2}\right)-y_{2}\left(1-y_{1}\right)\left(1-\kappa^{2} y_{1}\right)\right\}^{2} \\
& =\left\{y_{1}-y_{2}-\kappa^{2}\left(y_{1}^{2} y_{2}-y_{1} y_{2}^{2}\right)\right\}^{2} \\
& =\left(y_{1}-y_{2}\right)^{2} A\left(y_{1}, y_{2}\right)^{2} .
\end{aligned}
$$

By the definition of $a$

$$
\left\{A(z, w)^{2} a-B(z, w)\right\}^{2}-4 \lambda^{-2}\left(w^{\prime}\right)^{2}\left(z^{\prime}\right)^{2}=0 .
$$

Since $w$ and $z$ are solutions of $T=0$ and (1), the left hand side is

$$
\begin{aligned}
\{A(z, & \left.w)^{2} a-B(a, w)\right\}^{2}-4 S(w) S(z) \\
& =A(z, w)^{4} a^{2}-2 A(z, w)^{2} B(z, w) a+B(z, w)^{2}-4 S(w) S(z) \\
& =A(z, w)^{4} a^{2}-2 A(z, w)^{2} B(z, w) a+(z-w)^{2} A(z, w)^{2} \\
& =A(z, w)^{2}\left\{A(z, w)^{2} a^{2}-2 B(z, w) a+(z-w)^{2}\right\} .
\end{aligned}
$$

Since $A(z, w) \neq 0$, we have an algebraic relation over $k_{1}$ between $a$ and $z$ :

$$
A(z, w)^{2} a^{2}-2 B(z, w) a+(z-w)^{2}=0 .
$$

The left hand side of (2) is

$$
\begin{aligned}
&\left.\left(1-\kappa^{2} z w\right)^{2} a^{2}-2 z(1-w)\left(1-\kappa^{2} w\right)+w(1-z)\left(1-\kappa^{2} z\right)\right\} a \\
&+(z-w)^{2} \\
&=a^{2}\left(\kappa^{4} z^{2} w^{2}-2 \kappa^{2} z w+1\right) \\
&-2 a\left[\kappa^{2} w z^{2}+\left\{\kappa^{2} w^{2}-2\left(1+\kappa^{2}\right) w+1\right\} z+w\right] \\
&+z^{2}-2 z w+w^{2} \\
&= z^{2}\left(\kappa^{4} a^{2} w^{2}-2 \kappa^{2} a w+1\right) \\
&-2 z\left[\kappa^{2} w a^{2}+\left\{\kappa^{2} w^{2}-2\left(1+\kappa^{2}\right) w+1\right\} a+w\right] \\
&+a^{2}-2 w a+w^{2} .
\end{aligned}
$$

Hence we have a relation equivalent to (2):

$$
A(\alpha, w)^{2} z^{2}-2 B(a, w) z+(a-w)^{2}=0 .
$$

Since $z$ is transcendental over $k_{1}, a$ is transcendental over $k_{1}$ and satisfies $\left[k_{1}(a, z): k_{1}(z)\right]=2$. For the discriminant of $(2)$ is $16 S(z) S(w)$ by (1). We have $k_{1}\langle z\rangle=k_{1}(a, z)$. We shall prove that $a$ is a constant (cf. [2, p. 805]). Let us take an element $\alpha$ of $\bar{k}$ such that $\alpha^{2}=4 / \lambda$ and define a new differentiation signed by the dot in $k_{1}\langle\alpha, z\rangle$ by $\dot{x}=\alpha x^{\prime}$. Then, 


$$
\begin{aligned}
& a=\left\{B(z, w)-2^{-1} \dot{w} \dot{z}\right\} / A(z, w)^{2}, \\
& (\dot{z})^{2}=4 S(z), \quad(\dot{w})^{2}=4 S(w) .
\end{aligned}
$$

In what follows, we denote $A(z, w)$ and $B(z, w)$ by $A$ and $B$ respectively for simplicity. Differentiating both sides of $(\dot{w})^{2}=4 S(w)$, we have $2 \dot{w} \ddot{w}=4 S_{w} \dot{w}$ and $\ddot{w}=2 S_{w}$ since $\dot{w} \neq 0$. Hence,

$$
\begin{aligned}
B_{z}- & \ddot{w} / 2=B_{z}-S_{w} \\
= & (1-w)\left(1-\kappa^{2} w\right)+w\left\{2 \kappa^{2} z-\left(1+\kappa^{2}\right)\right\} \\
& -\left\{3 \kappa^{2} w^{2}-2\left(1+\kappa^{2}\right) w+1\right\} \\
= & -2 \kappa^{2} w^{2}+2 \kappa^{2} w z \\
= & 2 \kappa^{2} w(z-w) .
\end{aligned}
$$

On the other hand

$$
\begin{aligned}
2 A_{z} B & -(\dot{w})^{2} A_{w} \\
& =-2 \kappa^{2} w B+4 \kappa^{2} w w(1-w)\left(1-\kappa^{2} w\right) \\
& =2 \kappa^{2} w\left\{2 z(1-w)\left(1-\kappa^{2} w\right)-B\right\} \\
& =2 \kappa^{2} w\left\{z(1-w)\left(1-\kappa^{2} w\right)-w(1-z)\left(1-\kappa^{2} z\right)\right\} \\
& =2 \kappa^{2} w(z-w) A .
\end{aligned}
$$

Therefore

$$
A\left(B_{z}-\ddot{w} / 2\right)=2 A_{z} B-(\dot{w})^{2} A_{w}=2 \kappa^{2} w(z-w) A .
$$

Similarly we have

$$
A\left(B_{w}-\ddot{z} / 2\right)=2 A_{w} B-(\dot{z})^{2} A_{z}=2 \kappa^{2} z(w-z) A .
$$

From the above equalities and (4)

$$
\begin{aligned}
A^{3} \dot{a}= & \dot{z}\left\{A\left(B_{z}-\ddot{w} / 2\right)-2 A_{z} B+(\dot{w})^{2} A_{w}\right\} \\
& +\dot{w}\left\{A\left(B_{w}-\ddot{z} / 2\right)-2 A_{w} B+(\dot{z})^{2} A_{z}\right\} \\
= & 0 .
\end{aligned}
$$

Hence, $\dot{a}=0$, and $a^{\prime}=0$.

Let $k_{2}$ denote $k_{1}(\alpha)$ and $b$ be an element of $k_{2}\langle z\rangle$ defined by

$$
b=\left\{A(a, w)^{2} z-B(a, w)\right\} /\left(\alpha w^{\prime}\right) .
$$

Then, we have $b^{2}=S(a)$. In fact from (1) and (3) we have

$$
\begin{aligned}
\left\{A(a, w)^{2} z-B(a, w)\right\}^{2} & =B(a, w)^{2}-(a-w)^{2} A(a, w)^{2} \\
& =4 S(a) S(w),
\end{aligned}
$$

and $\left(\alpha w^{\prime}\right)^{2}=4 S(w)$ since $w$ is a solution of $T=0$. Hence, $k_{2}\langle z\rangle=$ $k_{2}(a, b)$ because $\left[k_{2}\langle z\rangle: k_{2}(a)\right]=\left[k_{2}(a, b): k_{2}(a)\right]=2$ and $b \in k_{2}\langle z\rangle$. 
By Lemma 1, there exists a nonsingular solution $v$ of $\left(y^{\prime}\right)^{2}=4 S(y)$ such that the field of constants of $k_{2}\langle v\rangle$ is $k_{0}$. Since $\alpha$ is a constant,

$$
\text { trans. } \operatorname{deg} k^{*}(a) / k^{*}=\text { trans. } \operatorname{deg} k_{0}(a) / k_{0}=1 \text {, }
$$

where $k^{*}=k_{2}\langle v\rangle$ (cf. [2, p. 767]). Hence, $a$ is transcendental over $k^{*}$. Therefore, $z$ is transcendental over $k^{*}$ by (3).

Let us define an element $\zeta$ of $k^{*}\langle z\rangle$ by

$$
\zeta=\left\{B(a, v)+b v^{\prime}\right\} / A(a, v)^{2} .
$$

Matsuda [3] proved that $\zeta$ is a solution of $\left(y^{\prime}\right)^{2}=4 S(y)$ and $k^{*}\left(\zeta, \zeta^{\prime}\right)=k^{*}(a, b)$ : We may take elements $s_{i}, c_{i}, d_{i}(1 \leqq i \leqq 3)$ of $\Omega$ such that

$$
\begin{aligned}
& s_{1}^{2}=v, \quad c_{1}^{2}=1-v, \quad d_{1}^{2}=1-\kappa^{2} v, \quad s_{1}^{\prime}=c_{1} d_{1} ; \\
& s_{2}^{2}=a, \quad c_{2}^{2}=1-a, \quad d_{2}^{2}=1-\kappa^{2} a, \quad b=s_{2} c_{2} d_{2} ; \\
& s_{3}=\left(s_{1} c_{2} d_{2}+s_{2} c_{1} d_{1}\right)\left(1-\kappa^{2} s_{1}^{2} s_{2}^{2}\right)^{-1} ; \\
& c_{3}=\left(c_{1} c_{2}-s_{1} s_{2} d_{1} d_{2}\right)\left(1-\kappa^{2} s_{1}^{2} s_{2}^{2}\right)^{-1} ; \\
& d_{3}=\left(d_{1} d_{2}-\kappa^{2} s_{1} s_{2} c_{1} c_{2}\right)\left(1-\kappa^{2} s_{1}^{2} s_{2}^{2}\right)^{-1} .
\end{aligned}
$$

We shall prove that

$$
c_{3}^{2}=1-s_{3}^{2}, \quad d_{3}^{2}=1-\kappa^{2} s_{3}^{2}, \quad s_{3}^{\prime}=c_{3} d_{3} .
$$

In fact by the definitions

$$
c_{1}^{\prime}=-s_{1} d_{1}, \quad d_{1}^{\prime}=-\kappa^{2} s_{1} c_{1}, \quad c_{2}^{\prime}=d_{2}^{\prime}=0 .
$$

Since

$$
1-\kappa^{2} s_{1}^{2} s_{2}^{2}=c_{1}^{2}+s_{1}^{2} d_{2}^{2}=c_{2}^{2}+s_{2}^{2} d_{1}^{2}
$$

we have

$$
\begin{aligned}
(1- & \left.s_{3}^{2}\right)\left(1-\kappa^{2} s_{1}^{2} s_{2}^{2}\right)^{2} \\
& =\left(1-\kappa^{2} s_{1}^{2} s_{2}^{2}\right)^{2}-\left(s_{1} c_{2} d_{2}+s_{2} c_{1} d_{1}\right)^{2} \\
& =\left(c_{1}^{2}+s_{1}^{2} d_{2}^{2}\right)\left(c_{2}^{2}+s_{2}^{2} d_{1}^{2}\right)-\left(s_{1} c_{2} d_{2}+s_{2} c_{1} d_{1}\right)^{2} \\
& =c_{1}^{2} c_{2}^{2}+s_{1}^{2} s_{2}^{2} d_{1}^{2} d_{2}^{2}-2 s_{1} s_{2} c_{1} c_{2} d_{1} d_{2} \\
& =\left(c_{1} c_{2}-s_{1} s_{2} d_{1} d_{2}\right)^{2} .
\end{aligned}
$$

Hence, $c_{3}^{2}=1-s_{3}^{2}$. Similarly, we have $d_{3}^{2}=1-\kappa^{2} s_{2}^{3}$, since

$$
1-\kappa^{2} s_{1}^{2} s_{2}^{2}=d_{1}^{2}+\kappa^{2} s_{1}^{2} c_{2}^{2}=d_{2}^{2}+\kappa^{2} s_{2}^{2} c_{1}^{2} \text {. }
$$

We have $s_{3}^{\prime}=c_{3} d_{3}$ according to the following:

$$
\begin{aligned}
& \left(1-\kappa^{2} s_{1}^{2} s_{2}^{2}\right)^{2} s_{3}^{\prime} \\
& \quad=\left(1-\kappa^{2} s_{1}^{2} s_{2}^{2}\right)\left(s_{1} c_{2} d_{2}+s_{2} c_{1} d_{1}\right)^{\prime}
\end{aligned}
$$




$$
\begin{aligned}
& -\left(1-\kappa^{2} s_{1}^{2} s_{2}^{2}\right)^{\prime}\left(s_{1} c_{2} d_{2}+s_{2} c_{1} d_{1}\right) \\
= & \left(1-\kappa^{2} s_{1}^{2} s_{2}^{2}\right)\left(s_{1}^{\prime} c_{2} d_{2}+s_{2} c_{1}^{\prime} d_{1}+s_{2} c_{1} d_{1}^{\prime}\right) \\
& +2 \kappa^{2} s_{1} s_{1}^{\prime} s_{2}^{2}\left(s_{1} c_{2} d_{2}+s_{2} c_{1} d_{1}\right) \\
= & \left(1-\kappa^{2} s_{1}^{2} s_{2}^{2}\right)\left(c_{1} c_{2} d_{1} d_{2}-s_{1} s_{2} d_{1}^{2}-\kappa^{2} s_{1} s_{2} c_{1}^{2}\right) \\
& +2 \kappa^{2} s_{1} s_{2}^{2} c_{1} d_{1}\left(s_{1} c_{2} d_{2}+s_{2} c_{1} d_{1}\right) \\
= & c_{1} c_{2} d_{1} d_{2}-s_{1} s_{2} d_{1}^{2}-\kappa^{2} s_{1} s_{2} c_{1}^{2}-\kappa^{2} s_{1}^{2} s_{2}^{2} c_{1} c_{2} d_{1} d_{2} \\
& +\kappa^{2} s_{1}^{3} s_{2}^{3} d_{1}^{2}+\kappa^{4} s_{1}^{3} s_{2}^{3} c_{1}^{2}+2 \kappa^{2} s_{1}^{2} s_{2}^{2} c_{1} c_{2} d_{1} d_{2}+2 \kappa^{2} s_{1} s_{2}^{3} c_{1}^{2} d_{1}^{2} \\
= & c_{1} c_{2} d_{1} d_{2}+\kappa^{2} s_{1}^{2} s_{2}^{2} c_{1} c_{2} d_{1} d_{2} \\
& -s_{1} s_{2}\left(d_{1}^{2}+\kappa^{2} c_{1}^{2}-\kappa^{2} s_{1}^{2} s_{2}^{2} d_{1}^{2}-\kappa^{4} s_{1}^{2} s_{2}^{2} c_{1}^{2}-2 \kappa^{2} s_{2}^{2} c_{1}^{2} d_{1}^{2}\right) ;
\end{aligned}
$$

here

$$
\begin{aligned}
d_{1}^{2}+ & \kappa^{2} c_{1}^{2}-\kappa^{2} s_{1}^{2} s_{2}^{2} d_{1}^{2}-\kappa^{4} s_{1}^{2} s_{2}^{2} c_{1}^{2}-2 \kappa^{2} s_{2}^{2} c_{1}^{2} d_{1}^{2} \\
= & d_{1}^{2}\left(1-\kappa^{2} s_{1}^{2} s_{2}^{2}-\kappa^{2} s_{2}^{2} c_{1}^{2}\right) \\
& +\kappa^{2} c_{1}^{2}\left(1-\kappa^{2} s_{1}^{2} s_{2}^{2}-s_{2}^{2} d_{1}^{2}\right) \\
= & d_{1}^{2}\left\{1-\kappa^{2} s_{2}^{2}\left(s_{1}^{2}+c_{1}^{2}\right)\right\}+\kappa^{2} c_{1}^{2}\left\{1-s_{2}^{2}\left(\kappa^{2} s_{1}^{2}+d_{1}^{2}\right)\right\} \\
= & d_{1}^{2} d_{2}^{2}+\kappa^{2} c_{1}^{2} c_{2}^{2} .
\end{aligned}
$$

Hence,

$$
\begin{aligned}
& \left(1-\kappa^{2} s_{1}^{2} s_{2}^{2}\right)^{2} s_{3}^{\prime} \\
& \quad=c_{1} c_{2} d_{1} d_{2}+\kappa^{2} s_{1}^{2} s_{2}^{2} c_{1} c_{2} d_{1} d_{2}-s_{1} s_{2}\left(d_{1}^{2} d_{2}^{2}+\kappa^{2} c_{1}^{2} c_{2}^{2}\right) \\
& \quad=\left(c_{1} c_{2}-s_{1} s_{2} d_{1} d_{2}\right)\left(d_{1} d_{2}-\kappa^{2} s_{1} s_{2} c_{1} c_{2}\right),
\end{aligned}
$$

and we have $s_{3}^{\prime}=c_{3} d_{3}$.

By the definition of $\zeta$ we have irreducible equations over $k^{*}$ :

$$
\begin{aligned}
& A(a, v)^{2} \zeta^{2}-2 B(a, v)+(a-v)^{2}=0, \\
& A(\zeta, v)^{2} a^{2}-2 B(\zeta, v) a+(\zeta-v)^{2}=0,
\end{aligned}
$$

as we get (2) and (3). Hence, $k^{*}\left(\zeta, \zeta^{\prime}\right)=k^{*}(a, b)=k^{*}\left(z, z^{\prime}\right)$. For we have $\left[k^{*}\left(\zeta, \zeta^{\prime}\right): k^{*}(\zeta)\right]=\left[k^{*}(a, \zeta): k^{*}(\zeta)\right]=\left[k^{*}(a, \zeta): k^{*}(a)\right]=\left[k^{*}(a, b): k^{*}(a)\right]=2$ by above equalities.

We remark that the adopting of the $s, c$ and $d$ gives an expository verification of the identity $\left(\zeta^{\prime}\right)^{2}=4 S(\zeta)$ proved by Matsuda [3].

\section{REFERENCES}

1. E. R. Kolchin, Existence theorem connected with the Picard-Vessiot theory of homogeneous linear ordinary differential equations, Bull. Amer. Math. Soc., 54 (1948), 927-932.

2. Galois theory of differential fields, Amer. J. Math., 75 (1953), 753-824.

3. M. Matsuda, Algebraic differential equations of the first order free from parametric singularities from the differential-algebraic standpoint, to appear. 
4. J. F. Ritt, Differential algebra, Amer. Math. Soc. Colloq. Publ. Vol. 33, New York, 1950.

5. M. Rosenlicht, An analogue of L'Hospital's rule, Proc. Amer. Math. Soc., 37 (1973) 369-373.

Received November 24, 1976 and in revised form July 20, 1977.

Department of Mathematics

OSAKA UNIVERSITY

TOYONAKA, OSAKA 560, JAPAN 



\section{PACIFIC JOURNAL OF MATHEMATICS}

\section{EDITORS}

RICHARD ARENS (Managing Editor)

University of California

Los Angeles, California 90024

C. W. Curtis

University of Oregon

Eugene, OR 97403

C. C. MOORE

University of California

Berkeley, CA 94720

\section{J. DUGUNDJI}

Department of Mathematics University of Southern California Los Angeles, California 90007

R. Finn AND J. Milgram Stanford University Stanford, California 94305

ASSOCIATE EDITORS
E. F. BECKENBACH

B. H. NeUMANN

F. WOLF

K. YoshidA

\section{SUPPORTING INSTITUTIONS}

UNIVERSITY OF BRITISH COLUMBIA UNIVERSITY OF SOUTHERN CALIFORNIA CALIFORNIA INSTITUTE OF TECHNOLOGY STANFORD UNIVERSITY UNIVERSITY OF CALIFORNIA MONTANA STATE UNIVERSITY UNIVERSITY OF TOKYO UNIVERSITY OF NEVADA, RENO UNIVERSITY OF UTAH NEW MEXICO STATE UNIVERSITY OREGON STATE UNIVERSITY UNIVERSITY OF OREGON

WASHINGTON STATE UNIVERSITY UNIVERSITY OF WASHINGTON OSAKA UNIVERSITY 


\section{Pacific Journal of Mathematics}

\section{Vol. 74, No. $1 \quad$ May, 1978}

Gerald Arthur Anderson, Computation of the surgery obstruction groups

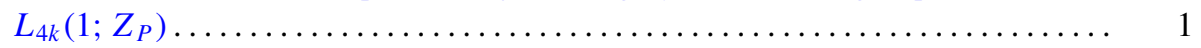

R. K. Beatson, The degree of monotone approximation ................ 5

Sterling K. Berberian, The character space of the algebra of regulated functions . . . 15

Douglas Michael Campbell and Jack Wayne Lamoreaux, Continua in the plane with

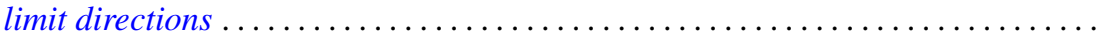

R. J. Duffin, Algorithms for localizing roots of a polynomial and the Pisot

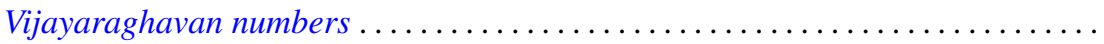

Alessandro Figà-Talamanca and Massimo A. Picardello, Functions that operate on

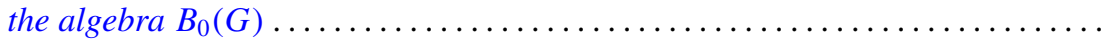

John Erik Fornaess, Biholomorphic mappings between weakly pseudoconvex

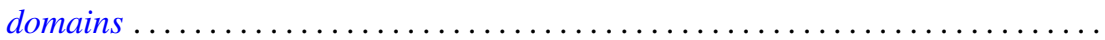

Andrzej Granas, Ronald Bernard Guenther and John Walter Lee, On a theorem of S.

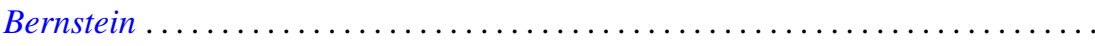

Jerry Grossman, On groups with specified lower central series quotients . .........

William H. Julian, Ray Mines, III and Fred Richman, Algebraic numbers, a constructive development . . . . . . . . . . . . . . . . . . . . . . .

Surjit Singh Khurana, A note on Radon-Nikodým theorem for finitely additive

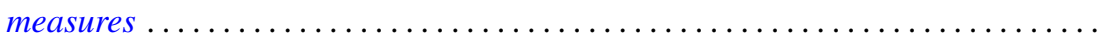

Garo K. Kiremidjian, A Nash-Moser-type implicit function theorem and nonlinear

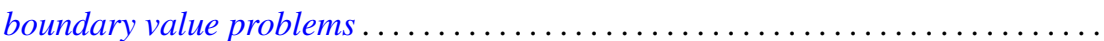

Ronald Jacob Leach, Coefficient estimates for certain multivalent functions ....

John Alan MacBain, Local and global bifurcation from normal eigenvalues. II . . 133

James A. MacDougall and Lowell G. Sweet, Three dimensional homogeneous algebras...

John Rowlay Martin, Fixed point sets of Peano continua ......

R. Daniel Mauldin, The boundedness of the Cantor-Bendixson order of some analytic sets...

Richard C. Metzler, Uniqueness of extensions of positive linear functions ..

Rodney V. Nillsen, Moment sequences obtained from restricted powers . .

Keiji Nishioka, Transcendental constants over the coefficient fields in differential elliptic function fields...

Gabriel Michael Miller Obi, An algebraic closed graph theorem

Richard Cranston Randell, Quotients of complete intersections by $\mathbf{C}^{*}$ actions . . 221

Bruce Reznick, Banach spaces which satisfy linear identities . .

Bennett Setzer, Elliptic curves over complex quadratic fields...

Arne Stray, A scheme for approximating bounded analytic functions on certain subsets of the unit disc.

Nicholas Th. Varopoulos, A remark on functions of bounded mean oscillation and bounded harmonic functions. Addendum to: "BMO functions and the

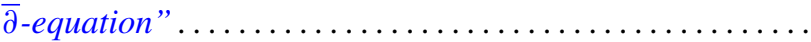

Charles Irvin Vinsonhaler, Torsion free abelian groups quasi-projective over their

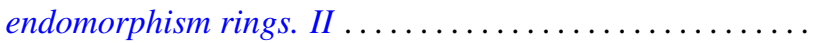

Thomas R. Wolf, Characters of $p^{\prime}$-degree in solvable groups ... 